\title{
Imputation of genotypes from different single nucleotide polymorphism panels in dairy cattle
}

\author{
T. Druet, ${ }^{*} \dagger^{1}$ C. Schrooten, $\ddagger$ and A. P. W. de Roos $¥$ \\ *Unit of Animal Genomics, Faculty of Veterinary Medicine and Centre for Biomedical Integrative Genoproteomics, University of Liège, \\ B-4000 Liège, Belgium \\ †Fonds de la Recherche Scientifique-FNRS, B-1000, Brussels, Belgium \\ ¥CRV, 6800 AL Arnhem, the Netherlands
}

\section{ABSTRACT}

Imputation of missing genotypes is important to join data from animals genotyped on different single nucleotide polymorphism (SNP) panels. Because of the evolution of available technologies, economical reasons, or coexistence of several products from competing organizations, animals might be genotyped for different SNP chips. Combined analysis of all the data increases accuracy of genomic selection or fine-mapping precision. In the present study, real data from 4,738 Dutch Holstein animals genotyped with custom-made 60K Illumina panels (Illumina, San Diego, CA) were used to mimic imputation of genotypes between 2 SNP panels of approximately 27,500 markers each and with 9,265 SNP markers in common. Imputation efficiency increased with number of reference animals (genotyped for both chips), when animals genotyped on a single chip were included in the training data, with regional higher marker densities, with greater distance to chromosome ends, and with a closer relationship between imputed and reference animals. With 0 to 2,000 animals genotyped for both chips, the mean imputation error rate ranged from 2.774 to $0.415 \%$ and accuracy ranged from 0.81 to 0.96 . Then, imputation was applied in the Dutch Holstein population to predict alleles from markers of the Illumina Bovine SNP50 chip with markers from a custom-made 60K Illumina panel. A crossvalidation study performed on 102 bulls indicated that the mean error rate per bull was approximately equal to $1.0 \%$. This study showed the feasibility to impute markers in dairy cattle with the current marker panels and with error rates below $1 \%$.

Key words: imputation, dairy cattle, genotyping

Received March 11, 2010.

Accepted July 8, 2010.

${ }^{1}$ Corresponding author: tom.druet@ulg.ac.be

\section{INTRODUCTION}

Genotype imputation consists of predicting missing genotypes. Many practical situations can lead to such missing genotypes. A few genotypes can be missing for technical reasons or because of inconsistencies with genotypes of related individuals. These cases should lead to small percentages of missing genotypes. Imputation is necessary with the use of tag SNP (e.g., Stephens and Scheet, 2005); to reduce costs, genotyping is performed only on some selected SNP and genotypes are predicted for the remaining SNP. People might also be interested in joining data from different experiments to perform a meta-analysis (e.g., Barrett et al., 2008) with increased power. Different genotyping panels might have been used in the initial experiments, resulting in large fractions of missing genotypes in the combined experiment. Imputation can be performed to impute SNP of very high density reference panels or sequence data (such as the International HapMap or 1000genomes projects) from smaller SNP panels genotypes (e.g., Marchini et al., 2007; Huang et al., 2009). In that case, the objective is to improve the efficiency of genome-wide association studies (Marchini et al., 2007; Servin and Stephens, 2007). Several methods have been proposed to perform genotype imputation in human populations (e.g., Stephens and Scheet, 2005; Marchini et al., 2007; Browning and Browning, 2009). All these methods rely on very large SNP density and linkage disequilibrium (LD).

For similar reasons, imputation is also important in dairy cattle populations. Indeed, exchange of data can result in higher accuracy of genomic selection or in improved power of QTL detection. VanRaden et al. (2009) showed that accuracy of genomic selection consistently increases with size of the training data set. In consequence, European cattle breeding organizations VIT (Verden, Germany), DHV (Bonn, Germany), UNCEIA (Paris, France), Viking Genetics (Randers, Denmark; Skara, Sweden; and Hollola, Finland), and CRV (Arnhem, the Netherlands) and their scientific partners decided to exchange genotypes from progeny-tested bulls 
to increase accuracy of their genomic selection program in a project called EuroGenomics. The EuroGenomics partners, however, had used different SNP panels: CRV had used 2 custom-made 60K Illumina panels (a first custom-made chip was designed in 2007 and a second was synthesized in 2008 with approximately 10,000 new markers), whereas the others had used the Illumina Bovine SNP50 (IB50K) chip (Illumina, San Diego, CA). In addition, breeders might be interested in using panels of different densities for different parts of the population (e.g., low-density panels for cows and high-density panels for bulls; Habier et al., 2009). New products such as the low-density panel Illumina Bovine $3 \mathrm{~K}$ and the high-density panel Illumina BovineHD will be available in 2010. It will be necessary to analyze jointly animals genotyped on the current SNP chips and animals that will be genotyped on these coming SNP chips.

The objective of the present study was to study imputation in dairy cattle with current marker panels and to apply imputation on the data of the EuroGenomics project because exchanged genotypes were obtained with 3 different $60 \mathrm{~K}$ bovine SNP chips. First, the feasibility of imputation on dairy cattle data sets with the method presented in Druet and Georges (2010) was investigated with a testing data set. Then, the same method was applied to the imputation of the markers of the IB50K chip for bulls genotyped with 1 CRV custom-made 60K Illumina panel for the EuroGenomics project.

\section{MATERIALS AND METHODS}

\section{Data}

Two data sets were used in this study. The first was used to test the feasibility of marker imputation between 2 distinct $60 \mathrm{~K}$ panels (test data), and the second consisted of a set of bulls genotyped on CRV $60 \mathrm{~K}$ chips for which imputation of IB50K SNP chip markers had been realized (EuroGenomics data). In the EuroGenomics project described above, some European dairy cattle breeding organizations and their scientific partners decided to exchange genotypes and pedigree of progeny-tested bulls. These were genotyped with one of 3 distinct marker panels: the IB50K chip, the CRV1 60K SNP chip (a custom-made 60K Illumina panel described in Charlier et al., 2008), and the CRV2 60K SNP chip (a new version of the CRV1 chip synthesized in 2008 with approximately 10,000 new markers, all present on the IB50K chip). Marker alleles specific to the IB50K chip needed to be imputed for bulls genotyped on the CRV chips and vice versa. In consequence, each partner would potentially benefit from higher
Table 1. Structure of the genotyped population in the test data

\begin{tabular}{lcc}
\hline $\begin{array}{l}\text { Animal } \\
\text { category }\end{array}$ & $\begin{array}{c}\text { No. of genotyped } \\
\text { offspring }\end{array}$ & $\begin{array}{c}\text { No. of } \\
\text { individuals }\end{array}$ \\
\hline Genotyped sires & $0^{1}$ & 146 \\
& 1 & 57 \\
& $2-5$ & 93 \\
& $6-10$ & 43 \\
Genotyped dams & $11-20$ & 26 \\
& 21 & 73 \\
& 1 & 62 \\
Genotyped offspring & $2-5$ & 56 \\
No genotyped parent & $6-10$ & 14 \\
Genotyped sire & $11-20$ & 9 \\
Genotyped dam & 21 & 3 \\
Both parents genotyped & 0 & 159 \\
\hline
\end{tabular}

${ }^{1}$ These are genotyped sires without genotyped offspring in the data set.

density marker maps resulting from the combination of different marker panels. The present study focused on imputation of marker alleles specific to the IB50K chip for a subset of Dutch Holstein bulls genotyped on a CRV chip. This imputation was realized before data exchange.

For both data sets, markers were filtered for HardyWeinberg equilibrium or minor allelic frequencies (higher than 0.0025), and markers for which less than $80 \%$ of animals had genotypes were removed. Offspring incompatible with their parent were first eliminated. Genotype data were then checked for inconsistencies between parent and offspring. Inconsistent genotypes were set missing.

In the test data, 4,738 animals were genotyped for the CRV1 chip. In total, 45,836 markers distributed over the autosomal chromosomes were used. The pedigree file, containing the genotyped animals and their ancestors, included 13,163 individuals. Table 1 gives information on the structure of the genotyped population. Genotyped sires and dams had on average 15.4 and 3.6

Table 2. Number of markers in common between SNP panels used in the EuroGenomics project ${ }^{1}$

\begin{tabular}{llll}
\hline SNP chip $^{2}$ & \multicolumn{1}{c}{ CRV1 } & \multicolumn{1}{c}{ CRV2 } & IB50K \\
\hline CRV1 & $46,947(2,974)$ & $41,618(68)$ & $10,954(529)$ \\
CRV2 & & $50,448(3,123)$ & $19,784(445)$ \\
IB50K & & & $45,182(974)$ \\
\hline
\end{tabular}

${ }^{1}$ Number of bulls genotyped on each chip is given in parentheses.

${ }^{2} \mathrm{CRV} 1$ = a custom-made $60 \mathrm{~K}$ Illumina panel (Illumina, San Diego, CA); CRV2 = a new version of the CRV1 chip synthesized in 2008 with approximately 10,000 new markers; IB50K = the Illumina Bovine SNP50 chip. 
Table 3. Number of markers per chip for the 3 different definitions of marker panels ${ }^{1}$

\begin{tabular}{lrrr}
\hline Chip 1 & Chip 2 & Common & Total \\
\hline 27,539 & 27,562 & 9,265 & 45,836 \\
27,651 & 27,735 & 20,011 & 35,375 \\
23,197 & 22,639 & 0 & 45,836 \\
\hline
\end{tabular}

${ }^{1}$ Markers were assigned to 2 virtual chips (chip 1 and chip 2). Markers common to the Illumina Bovine SNP50 chip and the custom-made 60K Illumina panel (Illumina, San Diego, CA) were conserved on both virtual chips, and remaining markers were randomly assigned to chip 1 or chip 2, thus resulting in 2 chips of approximately 27,500 SNP markers with 9,265 markers in common.

genotyped offspring, respectively. Up to 6 generations separated genotyped animals.

In the EuroGenomics data, 6,029 bulls were genotyped for one of the 2 CRV 60K SNP chips and 974 of these bulls were also genotyped for the IB50K chip (these bulls were either already genotyped on both chips or selected on number of genotyped descendents in the population). The number of bulls genotyped on each chip is presented in Table 2. A set of 81,175 markers $(19,784$ common markers, 25,398 IB50K markers, and 35,993 CRV markers) was conserved for the EuroGenomics project (Table 2). Slightly different sets of markers from the CRV1 chip were selected in both data sets. The pedigree file contained 16,874 animals.

\section{Method}

Imputation Scenarios Studied with the Test Data. To test the efficiency of genotype imputation, markers were assigned to 2 virtual chips (chip 1 and chip 2). Because testing the possibility to impute genotypes from the CRV1 chip to the IB50K chip was one of our goals, markers common to the IB50K chip and the CRV1 chip were conserved on both virtual chips and remaining markers were randomly assigned to chip 1 or chip 2, thus resulting in 2 chips of approximately 27,500 SNP markers with 9,265 markers in common (Table 3).

Genotyped animals were then separated in 3 groups: animals genotyped on both chips (reference individuals), animals genotyped on chip 1, and animals genotyped on chip 2. Designs with animals in each group or without any animals genotyped for chip 2 alone were tested. In both cases, imputation was studied with 0 to 2,000 reference individuals ( 8 sizes of reference sets were tested), resulting in total in 15 different designs (see Table 4 for the number of animals genotyped on each chip in each design). Bulls were ranked according to the expected number of copies of their haplotypes transmitted to the genotyped CRV population. These contributions were counted only if no other bull on the path to the progeny was genotyped. Only 438 bulls had a score higher than 0 . These bulls were preferentially selected as reference animals. Then, 144 dams with genotyped offspring were included in the reference group. Finally, additional reference animals were chosen randomly. The 2,738 individuals that were never selected as reference individuals were randomly assigned to the 2 groups genotyped on a single chip.

In addition, to study the effect of number of common markers between panels, the same designs were tested with different marker panels. Chip 1 and chip 2 were defined with approximately 27,500 SNP each but with 20,000 markers in common or with 22,500 SNP each and no markers in common (Table 3 ).

Marker Imputation. The method used in this study is described in detail in Druet and Georges (2010). The method models each chromosome independently. The physical map was converted to a genetic map assuming that $1 \mathrm{Mb}$ equals $1 \mathrm{cM}$. Haplotypes were first partially

Table 4. Mean imputation error rates and accuracy for different designs when chip 1 and chip $2^{1}$ have approximately 10,000 SNP in common

\begin{tabular}{|c|c|c|c|c|c|c|c|c|}
\hline \multirow[b]{2}{*}{$\begin{array}{l}\text { No. of } \\
\text { reference } \\
\text { individuals }\end{array}$} & \multicolumn{4}{|c|}{ Genotyped on chip 1 , chip 2 , or both } & \multicolumn{4}{|c|}{ Genotyped on chip 1 or on both chip 1 and chip 2} \\
\hline & $\begin{array}{l}\text { Genotyped } \\
\text { on chip } 1\end{array}$ & $\begin{array}{l}\text { Genotyped } \\
\text { on chip } 2\end{array}$ & $\begin{array}{l}\text { Mean } \\
\text { imputation } \\
\text { error rate } \\
(\%)\end{array}$ & $\begin{array}{c}\text { Mean } \\
\text { imputation } \\
\text { accuracy } \\
\left(\mathrm{r}^{2}\right)\end{array}$ & $\begin{array}{l}\text { Genotyped } \\
\text { on chip } 1\end{array}$ & $\begin{array}{l}\text { Genotyped } \\
\text { on chip } 2\end{array}$ & $\begin{array}{l}\text { Mean } \\
\text { imputation } \\
\text { error rate } \\
(\%)\end{array}$ & $\begin{array}{c}\text { Mean } \\
\text { imputation } \\
\text { accuracy } \\
\left(\mathrm{r}^{2}\right)\end{array}$ \\
\hline 0 & 3,351 & 1,387 & 2.774 & 0.811 & 4,738 & 0 & - & - \\
\hline 125 & 3,226 & 1,387 & 1.372 & 0.889 & 4,613 & 0 & 2.763 & 0.802 \\
\hline 750 & 2,601 & 1,387 & 0.555 & 0.949 & 3,988 & 0 & 0.742 & 0.935 \\
\hline 1,000 & 2,351 & 1,387 & 0.508 & 0.955 & 3,738 & 0 & 0.660 & 0.942 \\
\hline 1,500 & 1,851 & 1,387 & 0.449 & 0.959 & 3,238 & 0 & 0.560 & 0.951 \\
\hline 2,000 & 1,351 & 1,387 & 0.415 & 0.963 & 2,738 & 0 & 0.499 & 0.955 \\
\hline
\end{tabular}

${ }^{1}$ Markers were assigned to 2 virtual chips (chip 1 and chip 2). Markers common to the Illumina Bovine SNP50 chip and the custom-made 60K Illumina panel (Illumina, San Diego, CA) were conserved on both virtual chips, and remaining markers were randomly assigned to chip 1 or chip 2 , thus resulting in 2 chips of approximately 27,500 SNP markers with 9,265 markers in common. 
reconstructed based on genotypes of relatives (based on Mendelian segregation rules and linkage information) with the program LinkPHASE (Druet and Georges, 2010). Then, DAGPHASE (Druet and Georges, 2010) and Beagle (Browning and Browning, 2007) with scale and shift parameter set equal to 2.0 and 0.1 , respectively, were iteratively used to estimate the parameters of a directed acyclic graph (DAG). The programs were run first with genotypes from animals genotyped for all markers for 10 iterations and then with genotypes from all animals for 10 more iterations; this procedure improved imputation accuracy. As a consequence, all genotyped individuals were used in the training set. Haplotypes of reference individuals were then reconstructed with DAGPHASE. For all these steps, linkage information was ignored for parents with 5 or fewer offspring because Druet and Georges (2010) observed that use of this information increased haplotyping errors. Finally, in the last round, DAGPHASE was used to infer haplotypes and impute all missing markers of remaining individuals. The model implemented in Beagle can also be described as a hidden Markov model (Browning and Browning, 2007). Haplotype reconstruction and genotype imputation rely on the Viterbi algorithm (Rabiner, 1989), which predicts the most likely chain of hidden states. The forward-backward algorithm (Rabiner, 1989) was used to estimate the probability for the chain to be in hidden state $k$ at marker $m$. The posterior genotype probabilities were then estimated by summing the probabilities of the hidden states corresponding to the genotype.

Estimation of Imputation Accuracy. Imputation error rates were computed by marker and summarized genome-wide or per animal. The error rate was equal to the number of marker alleles incorrectly imputed divided by the total number of imputed marker alleles (twice the number of animals with missing genotype). The number of errors counted for an imputed genotype was equal to 0 when genotypes were identical, 1 if the real genotype was homozygous and the imputed genotype was heterozygous (or vice versa), and 2 if real and imputed genotypes were both homozygous but different.

Imputation accuracy was also estimated with the following per marker square correlation coefficient (Huang et al., 2009):

$$
r^{2}=\left(\frac{\sum_{i=1}^{n}\left(x_{i}-\bar{x}\right)\left(g_{i}-\bar{g}\right)}{\sqrt{\sum_{i=1}^{n}\left(x_{i}-\bar{x}\right)^{2} \sum_{i=1}^{n}\left(g_{i}-\bar{g}\right)^{2}}}\right)^{2},
$$

Journal of Dairy Science Vol. 93 No. 11, 2010 where $x_{i}$ denotes the imputed genotype for individual $i$, $\bar{x}$ denotes the mean value of the imputed genotypes $g_{i}$, and $\bar{g}$ represents these values for the true genotypes. The statistic is then averaged over all markers.

Comparison of Estimated and Observed Genotype Probabilities. Estimated posterior genotype probabilities for missing markers in the test data (with 1,000 reference individuals and without any animals genotyped for chip 2 alone) were compared with the real genotypes. For each imputed genotype, the probabilities of the 3 possible genotypes were estimated. Results were summarized for 100 genotype probability classes (from 0.00 to 1.00 by step of 0.01 ). For correct/ incorrect genotype, a correct/incorrect genotype was counted in the class corresponding to the associated genotype probability. Then, 2 ratios were computed for each genotype probability class: 1) the number of correct genotypes divided by the total number of genotypes in the class and 2) the number of correct alleles divided by the total number of alleles in the class (twice the number of genotypes).

Cross-Validation with the EuroGenomics Data Set. For imputation of IB50K marker alleles, correct genotypes are unknown. Therefore, to obtain some information on imputation accuracy, a cross-validation study was performed. Out of the 974 bulls that had genotypes for a CRV chip and the IBD50K chip, 190 bulls were selected based on 2 criteria: 1) they had no genotyped descendents (to conserve these bulls in the reference panel) and 2) their year of birth was between 1996 and 2003 (as the exchanged progeny-tested bulls). Then, 3 different sets of 34 validation bulls were drawn at random from these 190 bulls and the cross-validation was done separately for each set of bulls (by erasing their genotypes for the IB50K-specific markers) so that imputation error rates were estimated from approximately 100 bulls.

\section{RESULTS AND DISCUSSION}

\section{Estimation of Imputation Accuracy in the Feasibility Study}

The method used in this study predicts all missing marker alleles and leaves no missing markers. Moreover, allelic imputation error rate (proportion of incorrectly imputed marker alleles) was used rather than genotype imputation error rate (proportion of incorrect genotypes). When allelic imputation error rate is low and imputation on both haplotypes is relatively independent, genotypic imputation error rate can be approximated as twice the allelic imputation error rate. With 250 reference individuals or more, genotype imputation error rates ranged between 1.99 and 2.00 times the al-

Journal of Dairy Science Vol. 93 No. 11, 2010 
lelic imputation error rates (data not shown). With 125 reference individuals, this ratio was equal to 1.97 , still close to 2.00. Mechanically, genotype error rates cannot be higher than twice the allelic imputation error rate.

Imputation error rates are based on the sole imputed markers. After imputation, the proportion of incorrect marker alleles among all markers (genotyped and imputed markers) is equal to the fraction of imputed markers times the allelic imputation error rate plus the fraction of genotyped markers times 1.000 (assuming no genotyping errors). The same formula can be applied to accuracy of marker alleles. Therefore, the global accuracy of an individual is higher than the imputation accuracy and can easily be derived from the results presented in this study with the presented formula.

The mean imputation error rates for tested designs (with $~ 10,000$ SNP in common between chip 1 and chip 2 ) are presented in Table 4. Results are summarized for the 1,351 individuals always genotyped on chip 1 and for which markers of chip 2 are imputed to compare results for a fixed group of animals. Results are also expressed as mean imputation accuracies $\left(\mathrm{r}^{2}\right)$.

Imputation accuracy improved with number of reference individuals and in designs where some individuals were genotyped for chip 2 alone. With designs with 500 reference individuals or more, mean imputation error rates below $1 \%$ were obtained (accuracy above 0.91). In some designs, values below $0.5 \%$ were achieved (accuracy above 0.96). For comparison, when imputation is realized based on the most frequent genotypes or randomly (based on genotype frequencies), mean imputation error rates were equal to 19.85 and $26.77 \%$, respectively.

To get a better understanding of the effect of different factors on imputation efficiency, effects of marker density, distance to chromosome ends, and relationship between target and reference individuals were first analyzed in the design with 1,000 reference individuals and without any animals genotyped for chip 2 alone. Then the effect of general design was studied: number of reference individuals, number of common markers between SNP panels, and absence of individuals genotyped for chip 2 alone.

Marker Density. Because the DAG used in this study relies on LD between markers, higher marker density (hence higher LD between neighboring markers) should reduce imputation errors. In this study, the best results per chromosome were observed for chromosomes 6 and 14, which had one of the highest marker densities, and the worst results corresponded to chromosome 15 , the chromosome with the lowest marker density. Druet and Georges (2010) already observed increased imputation accuracy with higher density. Therefore, models describing imputation error rate per marker as a function of the marker density measured as the number of genotyped markers in the $1 \mathrm{Mb}$ segment centered at the imputed marker position $\left(\mathrm{d}_{1 \mathrm{Mb}}\right)$ were tested. $\mathrm{A}$ linear model describing the imputation error rate as a function of $\left(1+\mathrm{d}_{1 \mathrm{Mb}}\right)^{-1}$ was selected. The regression coefficient was highly significant $(P<0.0001)$ and was equal to $3.5 \%$. Figure 1 represents the estimated and observed relations between the imputation error rate and the marker density; each point corresponds to the observed mean error rate within a marker density class. The figure shows that when marker density increases from 0 to 80 genotyped markers within $1 \mathrm{Mb}$ of the imputed marker, the imputation error rates decrease by a factor of 10 (from 2.51 to $0.25 \%$ ), and that when marker density increases from 20 to 80 genotyped markers/ $\mathrm{Mb}$, the error rates decrease still. Therefore, even better imputation efficiency is expected with $60 \mathrm{~K}$ SNP chips and with the coming SNP panels of higher density. The slope of the curve decreased with marker density; the benefit of increasing marker density is much more important for low-density regions. For instance, from 0 to 2 markers/Mb, the imputation error rate decreased by $1.04 \%$, whereas from 20 to 72 markers/Mb, the imputation error rate decreased by $0.24 \%$.

Distance to Chromosome Ends. To obtain distance to chromosome ends, the marker position relative to each end of the map was counted and the smallest value was used. The selected quadratic polynomial model was highly significant $(P<0.0001)$. Figure 2 describes estimated and observed relationships; as for Figure 1, each point corresponds to the mean error rate within a distance class. Error rates were much higher at both ends of the chromosome, especially for markers in the first or last 10 positions of the chromosome. Mean imputation error rate for markers at the first or last position of the map was above $2.5 \%$, whereas for markers in the middle of the chromosome (after position 800 ) the mean error rate was below $0.5 \%$. The effect of distance to chromosome ends was stronger for the very beginning (or end) of the chromosome but was still visible for markers beyond the 600th position. Effect of distance can be explained by the fact that for markers at both extremes of the chromosome, no or few markers are at the left of the DAG (right for markers at the end of the chromosome). Marker imputation relies on surrounding markers, but for these markers, surrounding information is on only 1 side of the chromosome. In addition, the DAG allows for only a few paths at the beginning and the end of the chromosome and therefore regroups more haplotypes at these positions (with more variation). In the middle of the chromosome, long stretches of markers are available on both sides and the DAG uses information from many markers to identify different paths. Therefore, it regroups different 


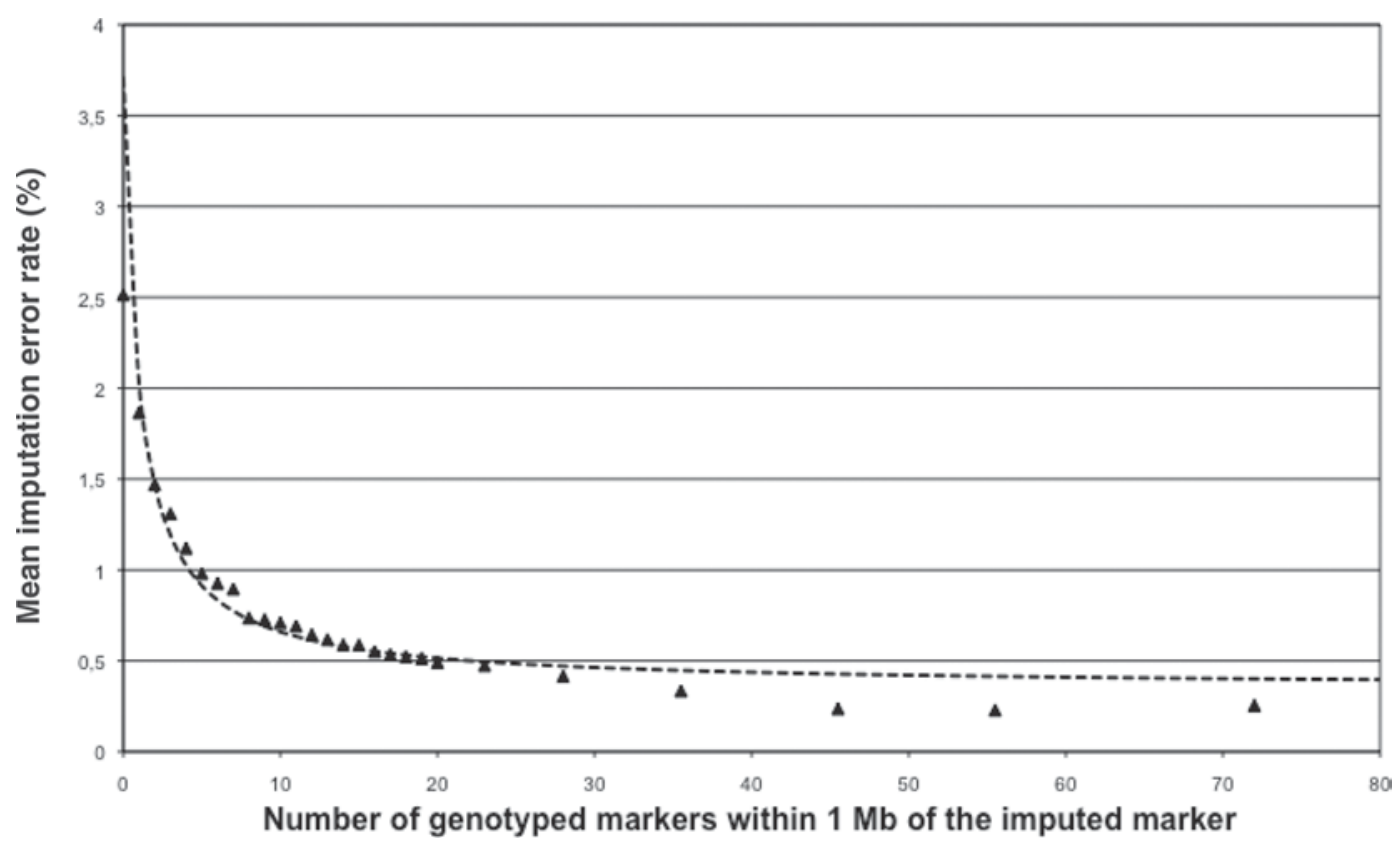

Figure 1. Relation between mean imputation error rate and marker density measured as the number of genotyped marker within $1 \mathrm{Mb}$ of the imputed marker. $\boldsymbol{\Delta}=$ Mean values observed for classes of marker density. Dashed curve indicates imputation error rate predicted by a linear model describing the error rate as a function of the inverse of the (density +1 ).

haplotypes more specifically. Biological reasons might also be responsible for the effect of marker position. Indeed, recombination rates (affecting linkage and LD and, therefore, imputation precision) are higher at both ends of the chromosomes (e.g., Chowdhury et al., 2007). To avoid confusion between effect of density and marker position, a model describing both effects jointly was fitted. Both were still significant $(P<0.0001)$. The determination coefficient of the model was low (0.081), indicating large residual terms. Errors in the genetic map (markers with incorrect positions or even from another chromosome) create large errors because these markers are difficult to impute because they are not in LD with the neighboring markers of the map. Some of these markers can eventually be detected based on LD and removed before imputation.

Relationship Between Target and Reference Individuals. The DAG models the observed haplotypes in a training data set; therefore, the relationship between animals with missing genotypes and the training set will affect the imputation error rate. A score based on ancestors of an animal that were reference animals was computed. The score represents the expected proportion of the genome of an animal that was inherited from a reference individual. The score is equal to the mean score of parents but when a parent is a reference individual, its score is replaced by 1.0. The computed scores ranged from 0.484375 to 1.0 , with the median equal to 0.9688 . This score only partially reflects the relationship between an animal and the training data because it ignores relatives that are not parents (progeny or sibs) and ignores relatives genotyped on a single chip. However, only reference animals had progeny and parents already contained an important amount of relatives' information. In addition, relatives genotyped on a single chip bring less information because it is more difficult to match their haplotypes with animals genotyped on the second chip. Therefore, the score estimated on ancestors that were reference animals indicates how well the haplotype is described in the DAG. It is important to remember that the method also directly uses linkage information to impute markers from progeny based on genotypes from parents. Furthermore, the DAG also indirectly models linkage: haplotypes from descendents will match haplotypes from their recent ancestors over relatively long fragments and they will be assigned to the same path over long stretches. Therefore, identification of the path of the descendents is more accurate and the imputation of missing genotypes is as well. Table 5 presents mean imputation error rate for animals grouped by score. The mean imputation error rate ranged from $1.16 \%$ for animals with a score below 0.75 to $0.14 \%$ for animals with both parents double genotyped. For groups of animals with intermediate scores, the imputation error rate consistently decreased as the score increased. A fitted linear regression between the mean imputation error rate and the score was highly significant $(P<0.0001)$. The extremely low imputation 


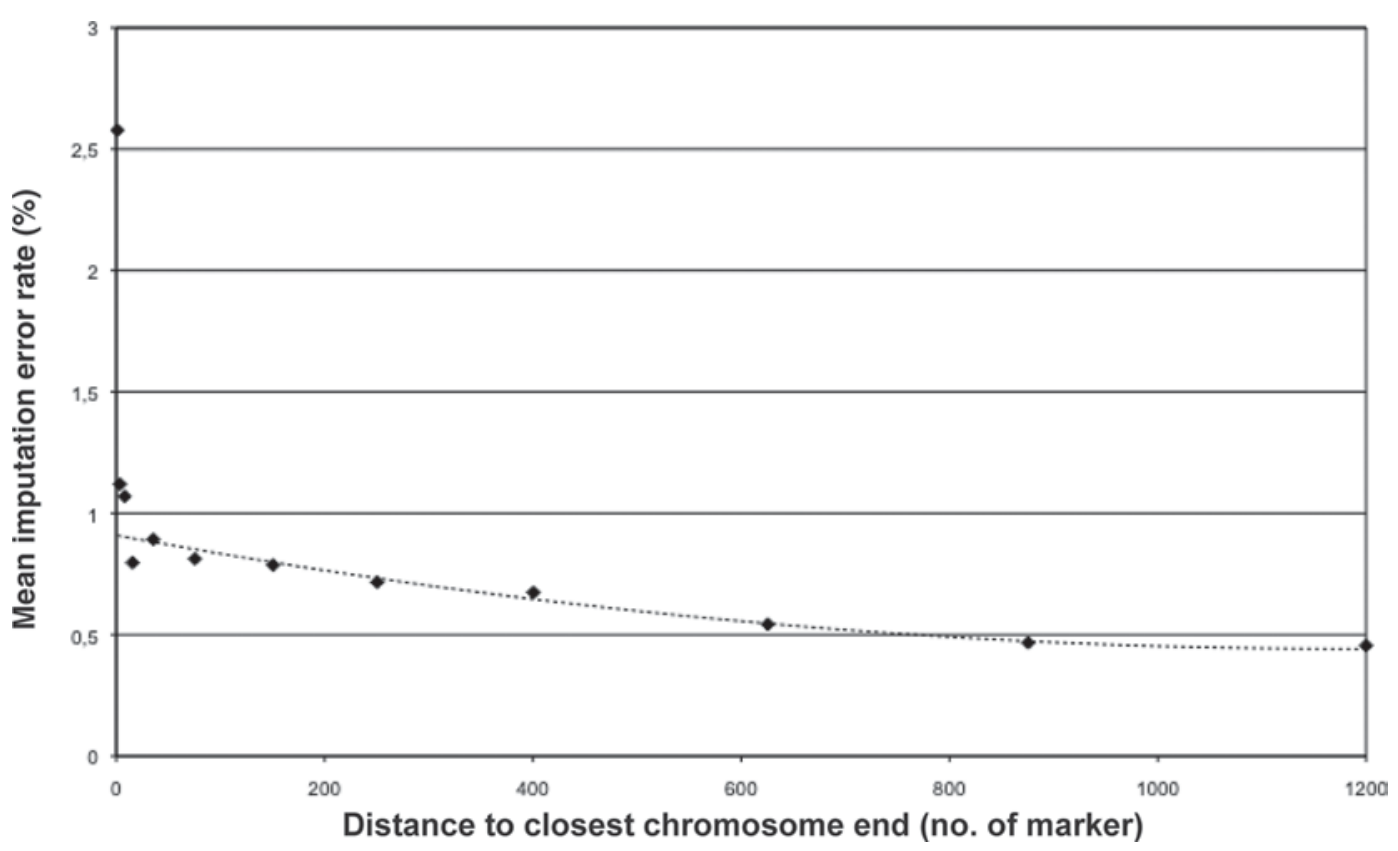

Figure 2. Relation between mean imputation error rate and distance to chromosome ends (counted from the closest end of the map). Mean values observed for classes of distance. Dotted curve indicates imputation error rate predicted by a linear model describing the error rate as a function of the position + position squared.

error rate for animals with both parents genotyped on all markers can be explained by the fact that for these animals, markers are imputed based on linkage only, whereas for other animals, alleles from 1 or 2 haplotypes are imputed based on LD.

Estimation of the DAG: Common Markers and Reference Individuals. Precise estimation of LD between markers (or parameters of the DAG) is required to optimally use that information and realize the imputation. The parameters need to be estimated between and within marker panels, and portions of the DAG

Table 5. Mean imputation error rates for animals grouped according to a score equal to the expected proportion of their genome inherited from reference individuals ${ }^{1}$

\begin{tabular}{lcc}
\hline Score & $\begin{array}{c}\text { No. of } \\
\text { animals }\end{array}$ & $\begin{array}{c}\text { Mean imputation } \\
\text { error rate }(\%)\end{array}$ \\
\hline$\leq 0.75$ & 33 & 1.16 \\
$0.75<$ score $\leq 0.90$ & 181 & 0.91 \\
$0.90<$ score $\leq 0.925$ & 105 & 0.80 \\
$0.925<$ score $\leq 0.95$ & 211 & 0.78 \\
$0.95<$ score $\leq 0.975$ & 325 & 0.71 \\
$0.975<$ score $<1.0$ & 259 & 0.68 \\
1 & 237 & 0.14 \\
\hline
\end{tabular}

${ }^{1}$ With 1,000 reference individuals and without any animals genotyped for chip 2 alone. Markers were assigned to 2 virtual chips (chip 1 and chip 2). Markers common to the Illumina Bovine SNP50 chip and the custom-made 60K Illumina panel (Illumina, San Diego, CA) were conserved on both virtual chips, and remaining markers were randomly assigned to chip 1 or chip 2 , thus resulting in 2 chips of approximately 27,500 SNP markers with 9,265 markers in common. specific to chip 1 or to chip 2 must be precisely connected. Through LD, common markers between different panels bring direct information to connect markers from both maps and therefore allow indirectly estimating the LD between markers from different chips. For these reasons, the number of common markers directly affects imputation accuracy. When few common markers are available, estimation of the DAG parameters can be obtained thanks to individuals genotyped on all markers. In this study, increasing the number of reference individuals improved imputation efficiency (Table 4). These individuals are very important because they allow estimating LD between markers from different panels but also between markers from the same panel. In addition, they bring information through linkage (see above).

Figure 3 presents mean imputation error rates for $27.5 \mathrm{~K}$ panels with approximately 10,000 or 20,000 markers in common and for a varying number of reference individuals (imputation error rates were estimated on the same set of markers). Importance of the 2 factors (number of common markers and number of reference individuals) is strongly dependent on the other factor. The benefit of having more common markers is higher with few reference individuals and vice versa. With fewer common markers, the curves describing the relation between mean imputation error rates and number of reference individuals are steeper. With high number of reference individuals, it is even better to have 


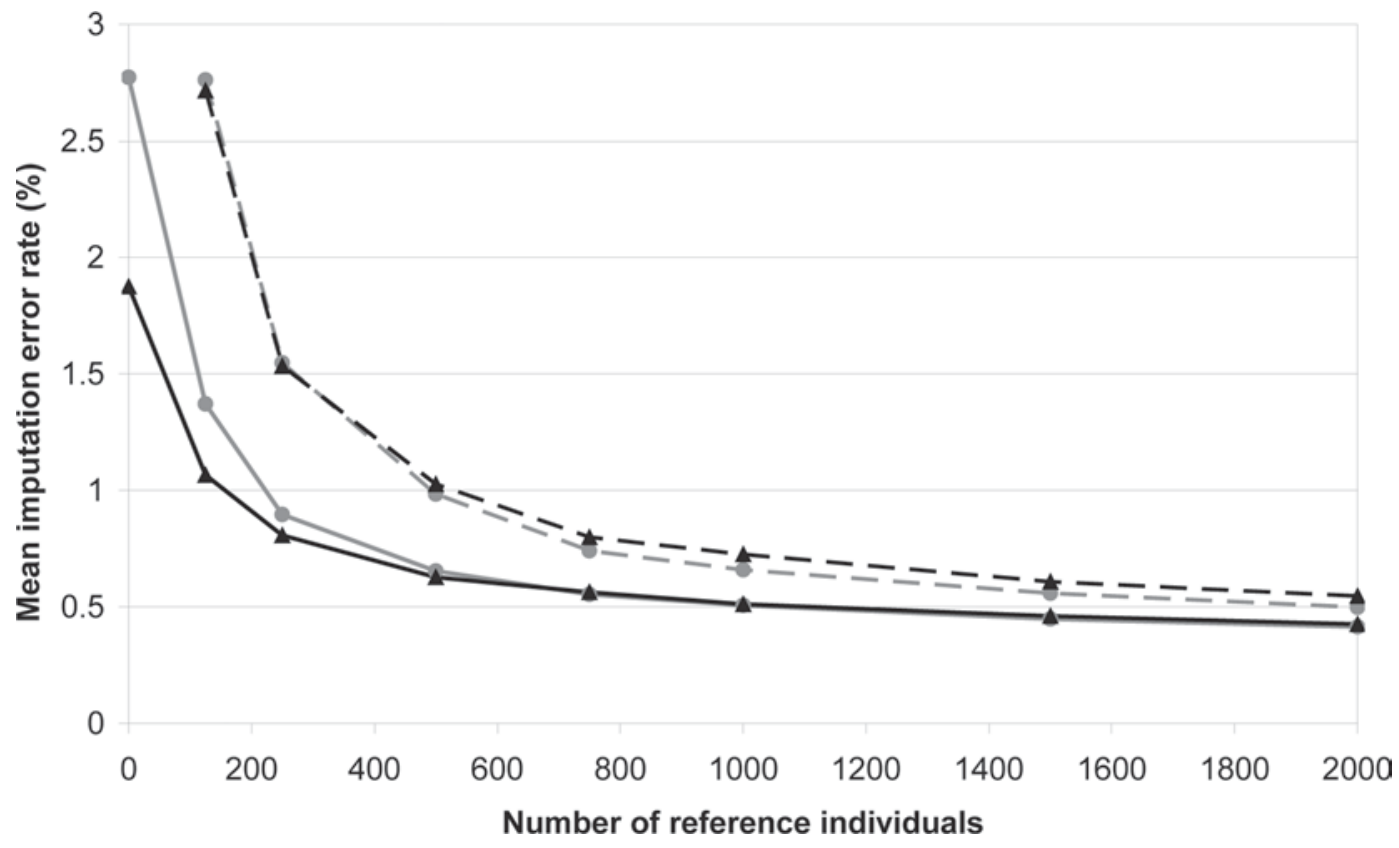

Figure 3. Relation between mean imputation error rate and number of reference individuals. Shown are designs with 20,000 (black triangles) or 10,000 (gray circles) common markers and designs with (solid lines) or without (dashed lines) animals genotyped for chip 2 alone. Markers were assigned to 2 virtual chips (chip 1 and chip 2). Markers common to the Illumina Bovine SNP50 chip and the custom-made 60K Illumina panel (Illumina, San Diego, CA) were conserved on both virtual chips, and remaining markers were randomly assigned to chip 1 or chip 2 , thus resulting in 2 chips of approximately 27,500 SNP markers with 9,265 markers in common.

fewer common markers. Indeed, reference individuals are genotyped for twice the number of markers on one chip minus the number of common markers. Therefore, with fewer common markers, reference individuals are genotyped for more markers. Depending on the number of reference individuals, the benefit of having these animals genotyped on more markers can be higher than the benefit of having more common markers.

As for marker density, the effect of the number of additional reference individuals was more pronounced for low number of reference animals (especially from 0 to 500). In our study, the first 500 reference individuals almost corresponded to all genotyped parents. The additional reference individuals improved marker allele prediction by bringing information through LD and linkage (they are collateral relatives) and by improving the reconstruction of the haplotypes of the parents. In all cases, the mean imputation error rate remained below $3 \%$. With (without) individuals genotyped for chip 2 alone, mean imputation error rates were below $1 \%$ with 250 (500) reference individuals or more.

The effect of the size of the reference set will depend on the structure of the target population. For a population presenting low variability, fewer reference individuals are required to achieve a given imputation accuracy because LD is higher and individuals derive from a smaller set of ancestors. When data from dif- ferent breeds are combined, it is expected that more reference individuals will be needed. Larger data sets might also require more reference individuals to obtain the same relationship between reference and target individuals.

When some individuals are genotyped for chip 2 alone, it is even possible to realize imputation without any reference individuals. However, this requires changing the imputation procedure: markers specific to chip 1 were first imputed for individuals genotyped on chip 2 (using only markers present on chip 1) and vice versa. Then, Beagle and DAGPHASE were run on all the markers. In the first iteration genotyped and imputed markers were used as input. This procedure was suggested by B. L. Browning and S. R. Browning (Department of Statistics, University of Auckland, Auckland, New Zealand; personal communication). The mean imputation error rate was equal to $2.77 \%$ with 10,000 markers in common and decreased to $1.88 \%$ with 20,000 markers in common, illustrating the effect of number of common markers.

Finally, Figure 4 illustrates that even without common markers it is possible to realize imputation based on reference individuals. The results were obtained with 2 chips containing 22,500 SNP each and are therefore not comparable with results obtained with $27,500 \mathrm{SNP}$ panels. 


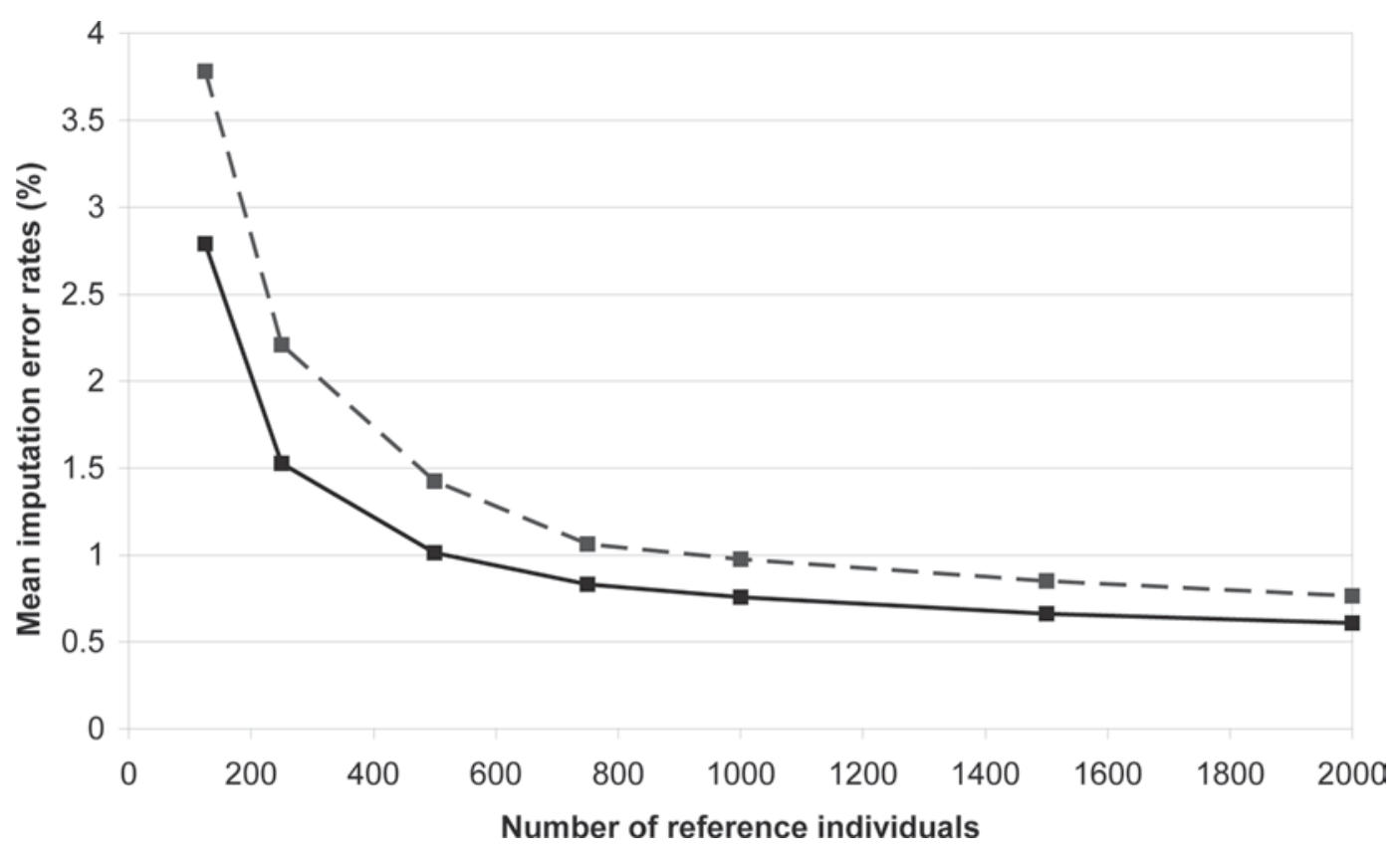

Figure 4. Relation between mean imputation error rate and number of reference individuals for designs without common markers. Shown are designs with (solid lines) or without (dashed lines) animals genotyped for chip 2 alone. Markers were assigned to 2 virtual chips (chip 1 and chip 2). Markers common to the Illumina Bovine SNP50 chip and the custom-made 60K Illumina panel (Illumina, San Diego, CA) were conserved on both virtual chips, and remaining markers were randomly assigned to chip 1 or chip 2, thus resulting in 2 chips of approximately 27,500 SNP markers with 9,265 markers in common.

Designs With or Without Animals Genotyped on Chip 2 Alone. Designs without any animals genotyped for chip 2 alone consistently resulted in higher imputation errors rates than designs with some individuals genotyped on chip 2 alone. This was observed in all configurations (Table 4, Figures 3 and 4). This decrease in imputation accuracy can be explained by the fact that, in the first case, LD or parameters of the DAG relative to markers specific to chip 2 (their association with common markers and with each other) are estimated thanks to the sole reference animals. In the second case, haplotypes of individuals genotyped for chip 2 alone are also used to estimate the parameters. As observed before, the benefit of additional information is higher when available information is low; having a set of animals genotyped for chip 2 alone to estimate the DAG improves more imputation accuracy when few reference individuals are available. With more markers in common, the benefit of this factor was also stronger (Figure 3).

Designs with animals genotyped on chip 1, chip 2, or both chips correspond to situations were data from different experiments (or populations) are merged together to increase power (for gene mapping or genomic selection) such as meta-analyses. Designs without any animals genotyped for chip 2 alone mimic imputation of markers from a large panel (all markers in our study) for individuals genotyped for a lower density panel (chip 1 in our study) because of a group of reference individuals genotyped for the larger panel. The lower density panel is then included in the larger panel. This situation is common in human genetics where, for instance, millions of SNP from the HapMap project available for a reference group of individuals are imputed for individuals genotyped for only a few hundred thousand markers. In dairy cattle, this situation will happen soon when imputation will be necessary to join data from animals genotyped on a 60K chip with animals genotyped on new chips with more than 600,000 markers. These results show that imputation is improved when all genotyped individuals are used rather than only reference individuals because animals genotyped on a subset of markers bring additional information.

\section{Genotype Probabilities}

The possibility to obtain posterior genotype probabilities in addition to imputed genotypes is desirable, especially when imputation accuracy is lower. The relationship between estimated genotype probability and genotype error rates was approximately linear with an intercept of 0 and a slope of 1 ; for a genotype probability of $\alpha$, the proportion of correct genotypes was equal to $\alpha$ (Figure 5 ). These results indicate that the genotype probabilities were correctly calibrated. 
Table 6. Imputation error rates estimated by cross-validation in the EuroGenomics project

\begin{tabular}{lccccc}
\hline $\begin{array}{l}\text { Genotyped } \\
\text { chip }^{1}\end{array}$ & $\begin{array}{c}\text { No. of } \\
\text { bulls }\end{array}$ & $\begin{array}{c}\text { No. of } \\
\text { markers }\end{array}$ & $\begin{array}{c}\text { No. of } \\
\text { common } \\
\text { markers }\end{array}$ & $\begin{array}{c}\text { No. of } \\
\text { imputed } \\
\text { markers }\end{array}$ & $\begin{array}{c}\text { Imputation } \\
\text { error rate (\%) }\end{array}$ \\
\hline CRV1 & 50 & 46,947 & 10,954 & 34,228 & 1.01 \\
CRV2 & 52 & 50,448 & 19,784 & 25,398 & 1.09 \\
Global & 102 & - & - & - & 1.05 \\
\hline
\end{tabular}

${ }^{1} \mathrm{CRV} 1$ = a custom-made 60K Illumina panel (Illumina, San Diego, CA); CRV2 = a new version of the CRV1 chip synthesized in 2008 with approximately 10,000 new markers.

When the error rates were based on the proportion of incorrect marker alleles, the relation remains linear but the proportion of correct marker alleles is approximately equal to $0.5+0.5 \times \alpha$. This indicates that when the genotype is wrong, only 1 marker allele out of 2 is incorrect. In the case of an additive genetic model, the marker allele error rate is more important than the genotype error rate.

\section{Imputation of IB50K Markers with CRV Markers for the EuroGenomics Project}

The efficiency of the imputation measured in the cross-validation study is based on a much smaller sample than that used in the first experiment. The overall mean imputation error rate per bull was equal to $1.05 \%$ (Table 6 ) and ranged from 0.28 to $2.98 \%$ per bull (median $0.96 \%$ ). Although the CRV2 chip contains more markers that are common with the IB50K chip than the CRV1 chip, the mean error rate was slightly lower for bulls genotyped on the CRV1 chip (1.01\%) than for bulls genotyped on the CRV2 chip (1.09\%). This can be explained by the fact that for animals genotyped on the CRV1 chip, IB50K-specific markers need to be imputed, as do markers in common between the CRV2 chip and the IB50K chip (but not on the CRV1 panel). For imputation of these markers, much more information is available because of the large number of common markers between CRV chips and the large number of bulls genotyped on both chips.

Although the observed error rates are higher than in the first study [the mean imputation error rate per animal (with 1,000 reference individuals and without any animals genotyped for chip 2 alone) was equal to $0.66 \%(\mathrm{SD}=0.347 \%)]$, they confirm that the imputed genotypes can be used to exchange progeny-tested bulls genotyped on different marker panels with approximately $1 \%$ genotype imputation error. The expected

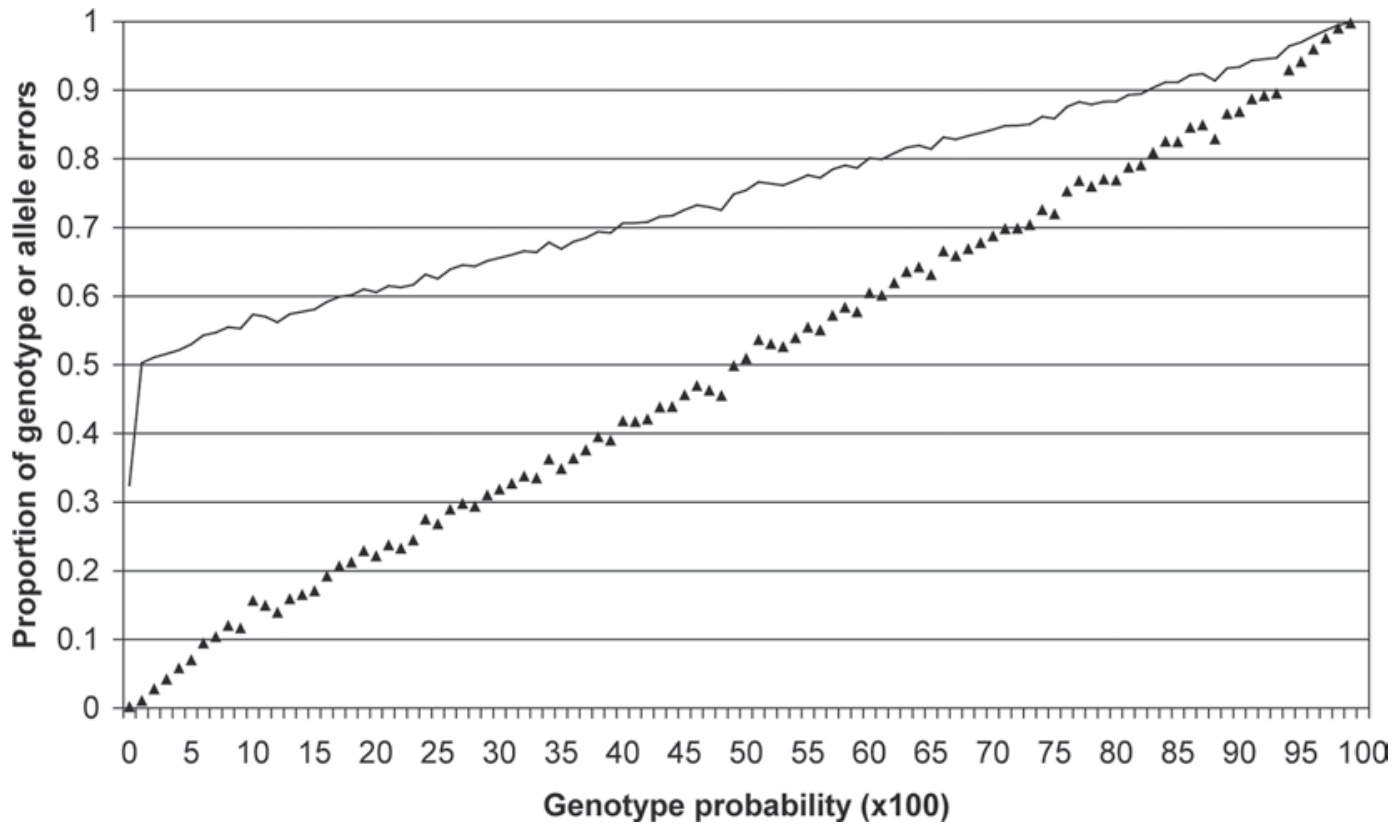

Figure 5. Relation between genotype probability class (by step of 1) and proportion of errors measured in genotypic errors ( $\mathbf{\Lambda}$ ) or allelic errors (solid line). 
proportion of the genome inherited from reference individuals was lower in the cross-validation study (range 0.2188 to 0.9766 , median 0.875 ) than in the feasibility study. None of the cross-validation bulls had both parents genotyped; in the feasibility study, it was shown that such animals decreased the overall error rate. This partially explains the lower imputation efficiency in the cross-validation study. Another factor might be related to the influence of $\mathrm{LD}$, which was shown to improve imputation efficiency. In the feasibility design, because markers were randomly assigned to virtual chips, more markers were imputed in high-density regions. This gave a higher weight to high-density regions. This bias is no longer present for the imputation of IB50K markers because the density of markers specific to the IB50K chip is not directly related to the density of the markers on the CRV chip. According to the results of the first experiment, adding genotypes from exchanged progeny-tested bulls genotyped on the IB50K chip will increase imputation precision. Consequences of using such imputed genotypes on genomic selection need to be studied and are under investigation by partners of the EuroGenomics project.

\section{Computational Requirements}

Computation times and memory requirements were measured on a computer cluster with Intel Xeon Harpertown L5420 at 2.50 Ghz (Intel Corp., Santa Clara, CA). Each chromosome was processed on its own central processing unit core. Times are indicative because they were estimated on a computing grid used by many concurrent users. For the test data, computational requirements are presented for a design with approximately 10,000 SNP in common and 1,000 reference individuals and without any animals genotyped for chip 2 alone. Computation times per chromosome ranged from $56 \mathrm{~min}$ to $5 \mathrm{~h} 41 \mathrm{~min}$, and memory requirements per chromosome ranged from 1.517 to 2.536 GB. For the EuroGenomics data, requirements were higher: from 2 h $27 \mathrm{~min}$ to $8 \mathrm{~h} 56 \mathrm{~min}$ and from 1.531 to 5.122 GB.

The current version of DAGPHASE has not been fully optimized for computational time and memory requirements. Memory requirements should be easy to reduce, whereas this would be more difficult for computational time. In addition, appropriate use of the program might maintain both values low. First, the DAG does not need to be estimated at every imputation and a reference training set might be used for this purpose (not the whole genotyped population). Second, when imputing a set of newly genotyped animals, only haplotypes of these animals and of their genotyped parents are needed. Running the imputation on such a subset would produce results much faster and require much less memory.

In the near future, new marker panels with probably more than 600,000 markers or even whole genome sequences of reference animals will be available. The results of the present study indicate that imputation of such huge marker panels from the current markers panels should result in less than $1 \%$ error rates because almost twice the number of markers will be available ( $\sim 45,000$ vs. $\sim 27,500$ in our study).

The same approach can also be used to impute markers of the current SNP panel with a lower density panel as suggested by Habier et al. (2009). This would allow screening the whole population at a lower cost. The efficiency of the present method in that case is under study, but higher error rates are expected because marker densities would be lower.

\section{CONCLUSIONS}

This study showed that, with a graphical model exploiting LD, it is feasible to efficiently impute missing genotypes with $60 \mathrm{~K}$ SNP chips. The efficiency was shown to increase with the number of reference individuals (genotyped on both chips), marker density, distance to chromosome ends, and expected proportion of the genome inherited from reference individuals. Addition of animals genotyped on a single chip was beneficial as well. For imputation of markers from 2 SNP panels, common markers or individuals genotyped on both panels are required to connect the graphs corresponding to each SNP panel. With two 27K SNP chips, only 10,000 SNP in common, and 1,000 out of 4,738 individuals genotyped for both chips, the imputation error rate was approximately $0.5 \%$, corresponding to an accuracy of 0.95 . The method was successfully applied to the imputation of IB50K marker alleles based on genotypes from the CRV chip. Finally, this imputation technique has been applied to genotypes of 16,000 Holstein bulls exchanged in the EuroGenomics project.

\section{ACKNOWLEDGMENTS}

Tom Druet is Research Associate from the Fonds de la Recherche Scientifique (Brussels, Belgium). The authors acknowledge the partners of the EuroGenomics project for their collaboration in this project. Sharon and Brian Browning are thanked for advice on imputation. The authors thank both reviewers for their helpful suggestions and corrections. This work was funded by grants of the Service Public de Wallonie (Jambes, Belgium) and from the Communauté Française de Belgique (Biomod ARC, Brussels, Belgium). The authors 
acknowledge the University of Liège (Liège, Belgium; SEGI and GIGA bioinformatics platform) for the use of NIC3 and GIGA-grid supercomputers.

\section{REFERENCES}

Barrett, J. C., S. Hansoul, D. L. Nicolae, J. H. Cho, R. H. Duerr J. D. Rioux, S. R. Brant, M. S. Silverberg, K. D. Taylor, M. M. Barmada, A. Bitton, T. Dassopoulos, L. W. Datta, T. Green, A. M. Griffiths, E. O. Kistner, M. T. Murtha, M. D. Regueiro, J. I Rotter, L. P. Schumm, A. H. Steinhart, S. R. Targan, R. J. Xavier, C. Libioulle, C. Sandor, M. Lathrop, J. Belaiche, O. Dewit, I. Gut, S. Heath, D. Laukens, M. Mni, P. Rutgeerts, A. Van Gossum, D. Zelenika, D. Franchimont, J. P. Hugot, M. de Vos, S. Vermeire, E. Louis, L. R. Cardon, C. A. Anderson, H. Drummond, E. Nimmo, T. Ahmad, N. J. Prescott, C. M. Onnie, S. A. Fisher, J. Marchini, J. Ghori, S. Bumpstead, R. Gwilliam, M. Tremelling, P. Deloukas, J. Mansfield, D. Jewell, J. Satsangi, C. G. Mathew, M. Parkes, M. Georges, and M. J. Daly. 2008. Genome-wide association defines more than 30 distinct susceptibility loci for Crohn's disease. Nat. Genet. 40:955-962.

Browning, B. L., and S. R. Browning. 2009. A unified approach to genotype imputation and haplotype-phase inference for large data sets of trios and unrelated individuals. Am. J. Hum. Genet. 84:210-223.

Browning, S. R., and B. L. Browning. 2007. Rapid and accurate haplotype phasing and missing-data inference for whole-genome association studies by use of localized haplotype clustering. Am. J. Hum. Genet. 81:1084-1097.

Charlier, C., W. Coppieters, F. Rollin, D. Desmecht, J. S. Agerholm, N. Cambisano, E. Carta, S. Dardano, M. Dive, C. Fasquelle, J. C. Frennet, R. Hanset, X. Hubin, C. Jorgensen, L. Karim, M.
Kent, K. Harvey, B. R. Pearce, P. Simon, N. Tama, H. Nie, S. Vandeputte, S. Lien, M. Longeri, M. Fredholm, R. J. Harvey, and M. Georges. 2008. Highly effective SNP-based association mapping and management of recessive defects in livestock. Nat. Genet. 40:449-454.

Chowdhury, R., P. R. J. Bois, E. Feingold, S. L. Sherman, and V. G. Cheung. 2009. Genetic analysis of variation in human meiotic recombination. PLoS Genet. 5:e1000648.

Druet, T., and M. Georges. 2010. A hidden Markov model combining linkage and linkage disequilibrium information for haplotype reconstruction and quantitative trait locus fine mapping. Genetics 184:789-798.

Habier, D., R. L. Fernando, and J. C. Dekkers. 2009. Genomic selection using low-density marker panels. Genetics 182:343-353.

Huang, L., Y. Li, A. B. Singleton, J. A. Hardy, G. Abecasis, N. A. Rosenberg, and P. Scheet. 2009. Genotype-imputation accuracy across worldwide human populations. Am. J. Hum. Genet. $84: 235-250$.

Marchini, J., B. Howie, S. Myers, G. McVean, and P. Donnelly. 2007. A new multipoint method for genome-wide association studies by imputation of genotypes. Nat. Genet. 39:906-913.

Rabiner, L. R. 1989. A tutorial on Hidden Markov Chains and selected applications in speech recognition. Proc. IEEE 77:257-286.

Servin, B., and M. Stephens. 2007. Imputation-based analysis of association studies: Candidate regions and quantitative traits. PLoS Genet. 3:e114.

Stephens, M., and P. Scheet. 2005. Accounting for decay of linkage disequilibrium in haplotype inference and missing-data imputation. Am. J. Hum. Genet. 76:449-462.

VanRaden, P. M., C. P. Van Tassell, G. R. Wiggans, T. S. Sonstegard, R. D. Schnabel, J. F. Taylor, and F. S. Schenkel. 2009. Invited review: Reliability of genomic predictions for North American Holstein bulls. J. Dairy Sci. 92:16-24. 